\title{
Ultrasound Poroelastic Tissue Typing
}

\author{
Piero Chiarelli ${ }^{1,2}$, Bruna Vinci ${ }^{1}$, Antonio Lanatà ${ }^{2}$, Valeria Gismondi ${ }^{2}$, Simone Chiarelli ${ }^{3}$ \\ ${ }^{1}$ National Council of Research of Italy, Pisa, Italy \\ ${ }^{2}$ Interdepartmental Research Center "E. Piaggio", Faculty of Engineering, \\ University of Pisa, Pisa, Italy \\ ${ }^{3}$ School of Engineering, University of Milan, Crema, Italy \\ E-mail:pchiare@ifc.cnr.it
}

Received October 4, 2011; revised November 12, 2011; accepted November 20, 2011

\begin{abstract}
Employing the poroelastic theory of acoustic waves in gels, the ultrasound (US) propagation in a gel medium filled by poroelastic spherical cells is studied. The equation of fast compressional wave, the phase velocity and the attenuation as a function of the elasticity, porosity and concentration of the cells into the gel matrix are investigated. The outcomes of the theory agree with the preliminary measurements done on PVA gel scaffolds inseminated by porcine liver cells at various concentrations. The feasibility of a non-invasive technique for the health assessment of soft biological tissues steaming by the model is analyzed.
\end{abstract}

Keywords: Ultrasound, Bi-Phasic Model of Living Tissues, Non-Invasive Ultrasound Assessment, Poroelastic Ultrasounds in Soft Tissues

\section{Introduction}

The present work is motivated in obtaining a reliable model for ultrasound (US) wave propagation in natural soft tissues [1-4].

In preceding works the authors validated a poro-elastic model for US propagation in synthetic as well as natural gels such as those of the extra-cellular matrix of soft tissues [4].

Even if the structure of a synthetic hydrogel is somehow different from that of a soft biological mesh, there exists a strong analogy between the macroscopic response of charged hydrogels and that of living tissues, such as for derma and cartilage [5], in the diffusional wave limit.

On the base of this analogy, the US bi-phasic model for soft tissues appears to be a promising tool for the development of non-invasive health assessment methods and for the study and the characterization of tissue mimicking phantom for US thermal therapy [6-8].

This fact is confirmed by the current research that clearly shows how the knowledge of the link between the poroelastic characteristics of a biological tissue and its acoustical behavior is a source of information that can be used for non-invasive investigations [8-10].

Nowadays, the US propagation in natural hydrogels, mostly composed of water, is usually modeled by means of the wave equation that holds for liquids [11].

This approach is poorly satisfying because it completely ignores the liquid-solid arrangement, and it does not completely explain the experimental behavior of the US propagation.

Moreover, there are many discrepancies between US propagation in water and in soft tissues both for transverse and longitudinal acoustic waves [1].

Actually, tissues are modeled as water solutions of natural polymers and proteins that may have bounded resonant states [1] with the US attenuation showing a frequency $(v)$ law [12]: $v^{(1+\delta)}$, with $\delta$ ranging between $1 / 4$ and $1 / 2 \quad(\delta=1$ for water $)$.

At high frequencies the classical poroelastic theories, mainly developed for geological studies, [13-22] lead to a dependence of attenuation with $\delta=1$ without any possibility to have a fractional value of $\delta$.

The recent gel bi-phasic model for US [4] shows that is possible to have a frequency dependence of attenuation with fractional values of $\delta$ as a direct consequence of the presence of bounded water onto the polymer network that affects the friction behavior of the fluid against the solid matrix.

At high frequencies the bounded water bearing lowers the friction between the free water and the polymer matrix leading to the peculiar behavior of the US phase velocity and attenuation of gels. 
Actually, the natural tissues are far to be homogenous but may present anisotropies, blood vessels and cells. In the present work spherical cells are introduced into the substrate of a homogeneous extra-cellular hydrogel matrix. The biological cells are designed as poro-elastic spheres, endowed by internal and superficial elasticity as well as permeability, and are assumed to be isotropically dispersed in the hydrogel environment. The model is developed in the continuum limit approach for US wavelength much bigger than the cells dimension (typically up to about $10 \mathrm{MHz}$ ).

The model presented here discloses how the behavior of the US propagation is linked to the arrangement of the biological medium and its poroelastic characteristics.

This papers shows that this fact can be used for the development of non-invasive health assessment techniques for tissues and organs by monitoring the elasticity, porosity and water content of the cells and the extracellular matrix. The detection of the liver cirrhosis is the short term application proposed in this paper [6].

\section{Poro-Elastic US Wave in Soft Living Tissues}

\subsection{US Wave in Highly Hydrated Gels}

The poroelastic wave equations for hydrogels can be obtained by introducing the appropriate fluid network interaction to take into account for the bounded water presence around the polymer chains [4].

Under the assumption that the bounded water volume fraction is very small and that the "polymer-bounded water aggregate" constitutes the solid matrix of the biphasic mean, it is possible to obtain with the following poroelastic motion equations [4]

$$
\begin{aligned}
\beta_{e} f \partial\left(e_{\alpha \alpha}^{*}-e_{\alpha \alpha}\right) / \partial t & =\eta_{(\omega)} \partial\left(\varepsilon_{\alpha \alpha}-e_{\alpha \alpha}^{*}\right) / \partial t \\
& +\kappa\left(\varepsilon_{\alpha \alpha}-e_{\alpha \alpha}^{*}\right) \\
\nabla^{2}\left(P \varepsilon_{\alpha \alpha}+Q e_{\alpha \alpha}\right)= & \partial^{2}\left(\rho_{11} \varepsilon_{\alpha \alpha}-\rho_{12} e_{\alpha \alpha}\right) / \partial t^{2} \\
& +\beta_{e} f \partial\left(e_{\alpha \alpha}^{*}-e_{\alpha \alpha}\right) / \partial t \\
\nabla^{2}\left(Q \varepsilon_{\alpha \alpha}+\operatorname{Re}_{\alpha \alpha}\right)= & \partial^{2}\left(\rho_{12} \varepsilon_{\alpha \alpha}-\rho_{22} e_{\alpha \alpha}\right) / \partial t^{2} \\
& -\beta_{e} f \partial\left(e_{\alpha \alpha}^{*}-e_{\alpha \alpha}\right) / \partial t \\
\beta_{e}= & \beta-\phi
\end{aligned}
$$

where $\varepsilon_{i j}$ is the solid strain tensor, $e_{\alpha \alpha}$ is the trace of the liquid strain tensor, $e_{\alpha \alpha}^{*}$ is the trace of the bounded water strain tensor; $P, Q$, and $R$ are the poroelastic medium constants that can be measured by means of jacketed and unjacketed experiments [18]; $\beta$ is the water volume fraction of the hydrogel, $\phi$ is the volume fraction of bounded water, $f$ is the inverse of the hydraulic permeability of the matrix [18], and $\rho_{11}, \rho_{12}$ and $\rho_{22}$, are the mass densities defined as: $\rho_{11}+2 \rho_{12}+\rho_{22}=\rho, \rho_{11}+\rho_{12}=\left(1-\beta_{e}\right) \rho_{\mathrm{s}}$, $\rho_{12}+\rho_{22}=\beta_{e} \rho_{f}$; where $\rho_{\mathrm{s}}$ and $\rho_{f}$ represent the solid and the liquid mass densities respectively, while $\rho$ is the total mass density of the biphasic medium. Moreover, the elastic constant $\kappa$ and the friction coefficient $\eta$ describe the polymer-bounded water interaction.

The above equations are derived by assuming that [4]:

1) The inertial effect of bounded water can be disregarded;

2) The trace of the strain tensor of the polymer $\varepsilon_{\alpha \alpha}$ approximates that one of the solid aggregate.

Moreover, by introducing the condition that the water content in the hydrogel is very high, further approximations can be introduced into the wave equations such as

$$
\begin{gathered}
R \gg Q \gg P \\
Q / R \cong\left(1-\beta_{e}\right) / \beta_{e} \cong\left(1-\beta_{e}\right) \\
\rho_{12}=-\rho_{11} \ll \rho_{22}
\end{gathered}
$$

to obtain

$$
\begin{gathered}
\nabla^{2}\left(\operatorname{Re}_{\alpha \alpha}\right)=\beta_{e}^{2} \partial^{2}\left(\beta_{e} \rho_{f} e_{\alpha \alpha}\right) / \partial t^{2} \\
+\beta_{e} \partial^{2} \rho_{11}\left(\varepsilon_{\alpha \alpha}-e_{\alpha \alpha}\right) / \partial t^{2} \\
f \partial\left(e_{\alpha \alpha}^{*}-e_{\alpha \alpha}\right) / \partial t \cong(Q / \mathrm{R}) \partial^{2}\left(\beta_{e} \rho_{f} e_{\alpha \alpha}\right) / \partial t^{2} \\
\beta_{e} f \partial\left(e_{\alpha \alpha}^{*}-e_{\alpha \alpha}\right) / \partial t=\eta_{(\omega)} \partial\left(\varepsilon_{\alpha \alpha}-e_{\alpha \alpha}^{*}\right) / \partial t \\
+\kappa\left(\varepsilon_{\alpha \alpha}-e_{\alpha \alpha}^{*}\right)
\end{gathered}
$$

For the fast plane wave $\varepsilon_{\alpha \alpha}=C e^{\alpha x} e^{i(k x-\alpha t)}$ Equation (1a) reads

$$
\beta_{e} f \partial\left(e_{\alpha \alpha}^{*}-e_{\alpha \alpha}\right) / \partial t=F_{(\omega)} \partial\left(\varepsilon_{\alpha \alpha}-e_{\alpha \alpha}\right) / \partial t
$$

where the complex friction coefficient of the gel $F(\omega)$ reads

$$
F(\omega)=\left[\left(\beta_{e} f\right)^{-1}+(\eta(\omega)+\mathrm{j}(\kappa / \omega))^{-1}\right]^{-1}
$$

Leading, by Equation (6a), to the characteristic equation

$$
(k+i \alpha)^{2}=\omega^{2} \rho_{f}\left(\beta_{e}^{3} / R\right)\left(1-i \omega\left(1-\beta_{e}\right) \rho_{11} / F_{(\omega)}\right)
$$

that transformed In two real equations in $\alpha$ and $c$ gives

$$
\begin{array}{r}
c^{2}=c_{0}^{2}\left(1+\alpha^{2} / k^{2}\right) /\left(1+\beta_{e}^{-2}\left(1-\beta_{e}\right) \omega \rho_{11} \operatorname{Im}\left\{F_{(\omega)}^{-1}\right\}\right) \\
2 a / k=-\left(c / c_{0}\right)^{2} \omega\left(1-\beta_{e}\right) \rho_{11} \operatorname{Re}\left\{F_{(\omega)}^{-1}\right\}
\end{array}
$$

where

$$
c_{0} \cong \frac{c_{f}}{(\beta-\varphi)^{3 / 2}}
$$

is the pure elastic longitudinal US wave velocity in the 
gel, $c_{f}=\left(R / \rho_{f}\right)^{1 / 2}$ the US velocity in the intermolecular fluid (free water) and

$$
\begin{aligned}
\operatorname{Re}\left\{F^{-1}\right\}= & {\left[\eta\left(1+\eta / \beta_{e} f\right)+\left(\kappa^{2} / \beta_{e} f \omega^{2}\right)\right] /\left(\eta^{2}+(\kappa / \omega)^{2}\right) } \\
& \operatorname{Im}\left\{F^{-1}\right\}=-(\kappa / \omega) /\left(\eta^{2}+(\kappa / \omega)^{2}\right)
\end{aligned}
$$

Assuming that the polymer-bounded water viscosity $\eta(\omega)$ follows the frequency behavior [4]

$$
\eta_{(\omega)}=\eta_{0}\left(\omega_{g} / \omega\right)^{\delta}
$$

with $0<\delta \leq 1 / 2$ and where $\omega_{g}=2 \pi \eta_{0} / \rho_{f}$, it follows that

$$
\begin{gathered}
\lim _{\omega / \omega_{\mathrm{g}} \gg 1} \operatorname{Re}\left\{F^{-1}\right\} \cong 1 / \eta_{(\omega)} \\
\lim _{\omega / \omega_{\mathrm{g}} \gg 1} \operatorname{Im}\left\{F^{-1}\right\} \cong 0
\end{gathered}
$$

Thence, in a hydrogel with a infinitely dilute polymer matrix $\left(\beta_{e} \approx 1\right.$ and $\left.\rho_{11} \ll 1\right)$ Equations (10) and (11) read

$$
c^{2}=c_{0}^{2}\left(1+\alpha^{2} / k^{2}\right) \cong c_{0}^{2}
$$

where $c \cong c_{0}^{2}$ since typically $(\alpha / k)^{2}$ is very small (of order of $10^{-3}$ in hydrogels), and

$$
2 \alpha / k=-\left(c / c_{0}\right)^{2}(\omega / \omega)^{1+\delta}(1-\beta+\varphi) \sigma_{p f}
$$

where

$$
\sigma_{p f}=2 \pi \rho_{11} / \rho_{f}
$$

Finally, when the polymer network is not very diluted, but has a real finite concentration, the series expansion as a function of the fractional polymer volume $(1-\beta)$ can be introduced into Equation (12) to read [4]

$$
c_{0}^{2} \cong \frac{c_{f}^{2}}{(\beta-\varphi)^{3}+\chi_{1}(1-\beta)+\chi_{2}(1-\beta)^{2}}
$$

\subsection{US Wave Equation in a Hydrogel with Dispersed Cells}

When we describe a tissue as a hydrogel biphasic mean containing cells, we have to refer to the overall tissue constants $\beta_{t}, \mathrm{R}_{\mathrm{t}}, \rho_{f t}, F_{t}$ and so on, into the Equations (6a)-(6c) that for plane waves lead to

$$
\nabla^{2}\left(c_{t 0} e_{\alpha \alpha}\right)=\partial^{2} e_{\alpha \alpha} / \partial t^{2}+\left(1-\beta_{t}\right) F^{-1} \rho_{11} \partial^{3} e_{\alpha \alpha} / \partial t^{3}
$$

where

$$
c_{t 0}=R / \rho_{f} \beta_{t}^{3}
$$

The constants $\beta_{t} R_{t}$ and $\rho_{f t}$ affect the phase velocity of the elastic limit, while $\beta_{t}$ and $F_{t}$ affect the US absorbance. As far as it concerns $\beta_{t}$, it influences the US attenuation through $\rho_{11}$ as it can be explicitly shown in diluted
Berryman matrices [23] for which $\rho_{11} \cong\left(1-\beta_{t}\right) \rho_{s}$.

By defining $\gamma$ as the fractional volume of cells inside the tissue to read

$\gamma=$ Total volume of cells/Total volume of tissue, the mean fractional water content of the tissue $\beta_{t}$ reads

$$
\beta_{t}=(1-\gamma) \beta_{e}+\gamma \beta_{c}=\beta_{e}\left\{1-\gamma\left(1-\beta_{c} / \beta_{e}\right)\right\}
$$

where $\beta_{c}$ is the fractional cells free water content.

\subsection{Us Speed in Natural Tissue}

In order to investigate the US phase velocity in the hydrogel-cells syncytium we need to determine the biphasic parameters concerning the inertial and the elastic terms in the motion Equations (22).

As far as it concerns the compressibility modulus $R_{t}$ and the mass density $\rho_{f t}$ of the syncytium they are influenced by the chemical (e.g., ionic strength) and mass composition of the cells that usually differ from that ones of the extra-cellular matrix. Thence, $c_{f t}=\left(R_{t} / \rho_{f t}\right)^{1 / 2}$ and the elastic phase velocity $c_{t 0}$ are function of cell concentration $\gamma$.

Assuming that the velocity $c_{f t}=R_{t} / \rho_{f t}$ slightly changes as a function of the cells concentration $\gamma$, the series expansion

$$
c_{f t}=\left(R_{t} / \rho_{f t}\right)^{1 / 2} \cong c_{f}\left\{1+A \gamma+A \gamma^{2}\right\}
$$

can be retained for the tissue.

Moreover, analogously to Equations (18) and (21) for a biological tissue we can assume

$$
c_{t}=c_{t 0}\left(1+\left(\alpha_{t} / k\right)^{2}\right)^{1 / 2} \cong c_{t 0} \cong c_{f t} / \beta_{t}^{3}
$$

and, hence,

$$
c_{t} \cong c_{f}\left\{1+A \gamma+A \gamma^{2}\right\} / \beta_{t}^{3}
$$

where $c_{t 0}$ is the pure elastic phase velocity in the tissue and $\alpha_{t}$ is the absorbing coefficient we are going to calculate in the next paragraph.

\subsection{Us Attenuation in Natural Tissue}

In this section we derive the complex friction coefficient $F(\omega)$ (the inverse of hydraulic conductance of the syncytium) that is responsible for the US attenuation.

We assume the following complex hydraulic conductance:

1) $F_{g(\omega)}^{-1} \cong \eta_{g}^{-1}$ for the extra-cellular hydrogel;

2) $F_{b(\omega)}^{-1} \cong \eta_{b}^{-1}$ for the internal jelly cell body;

3) $F_{m(\omega)}^{-1}$ for the cells membrane.

For sinusoidal inputs of frequency $\omega / 2 \pi$ we assume 
that

$$
F_{m(\omega)}^{-1}=K_{m}+i \omega b E_{m}^{-1}
$$

where the real part $K_{m}$ is the hydraulic permeability of the cell membrane and where the imaginary one is its superficial compliance proportional to the inverse of Young's elastic modulus $E_{m}$. In the case where the cell membrane thickness is much smaller than the cell diameter, $b^{-1}$ represents the membrane thickness.

Since the problem of sinusoidal electrical inputs has already been solved [24], we can easily find the overall conductance of the tissue by making use of the electrichydraulic analogy.

Given a syncytium of cells of radius " $a$ " embedded in a hydrogel with the following electrical parameters

1) $\sigma_{g}$ electrical conductance of the extra-cellular hydrogel;

2) $\sigma_{b}$ electrical conductance of the internal body of the cell;

3) $\sigma_{m}$ surface electrical conductance of the cells membrane;

4) $C_{m}$ surface electrical capacity of the cells membrane.

The global electrical admittance $Y$ of the syncytium [21] reads

$$
Y^{*}=\sigma_{g} \cdot \frac{\left\{2(1-\gamma) \sigma_{g}+(1+2 \gamma) \sigma_{b}\right\}}{\left\{(2+\gamma) \sigma_{g}+(1-\gamma) \sigma_{b m}\right\}}
$$

where

and where

$$
\sigma_{b m}=\sigma_{b} a Y_{m} /\left(\sigma_{b}+a Y_{m}\right)
$$

$$
Y_{m}=\sigma_{m}+i \omega C_{m}
$$

Therefore by mean of the following substitutions

1) $\sigma_{g} \rightarrow F_{g}^{-1}$ hydraulic admittance of the extra-cellular hydrogel;

2) $\sigma_{b} \rightarrow F_{b}^{-1}$ hydraulic admittance of the internal body of the cell;

3) $\sigma_{m} \rightarrow K_{m}$ surface hydraulic admittance of the cells membrane;

4) $C_{m} \rightarrow b E_{m}^{-1}$ surface elasticity of the cells membrane.

We end up with the global hydraulic admittance of cellular syncytium

$$
F^{-1}=F_{g}^{-1} \cdot \frac{\left\{2(1-\gamma) F_{g}^{-1}+(1+2 \gamma) F_{b}^{-1}\right\}}{\left\{(2+\gamma) F_{g}^{-1}+(1-\gamma) F_{b m}^{-1}\right\}}
$$

where $F_{b m}^{-1}$ is a combined bulk-membrane permeability that reads

$$
F_{b m}^{-1}=F_{b}^{-1} b a F_{m}^{-1} /\left(F_{b}^{-1}+a F_{m}^{-1}\right)
$$

In order to apply the above model to a biological tis- sue we need to single out the relative magnitude of the hydraulic constants.

Since the superficial membrane of the cells has a very low hydraulic permeability (it separates the inner cell body from the external hydrogel matrix), we expect that the frequency $\omega_{m} / 2 \pi$ is not very high.

Therefore, at high frequencies $\omega \gg \omega m=K m / b E_{m}^{-1}$, the compliance of the cell membrane prevails on its permeability and it follows that

$$
F_{m}^{-1}=K_{m}+i \omega b E_{m}^{-1} \cong i \omega b E_{m}^{-1}
$$

that

$$
F_{b m}^{-1}=F_{b}^{-1} /\left(1+F_{b}^{-1} / i \omega b a E_{m}^{-1}\right)
$$

and that the hydraulic admittance of the tissue reads

$$
F^{-1}=\eta_{g}^{-1} \cdot \frac{\left\{2(1-\gamma) \eta_{g}^{-1}+(1+2 \gamma) \eta_{b}^{-1}\right\}}{\left\{(2+\gamma) \eta_{g}^{-1}+(1-\gamma) \eta_{b}^{-1}\left(1+\eta_{b}^{-1} / \text { ai } \omega b E_{m}^{-1}\right)\right\}}
$$

If we assume $F_{b}^{-1}$ to have the typical form of Equations (15) and (16) to read

$$
F_{b}=\eta_{b(\omega)}=\eta_{0 b}\left(\omega_{g b} / \omega\right)^{\delta}
$$

with $\omega_{g b}=2 \pi \eta_{0 b} / \rho_{f b}$, it follows that

$$
F_{b m}^{-1}=\eta_{b}^{-1}\left(1+\eta_{b}^{-1} / a i \omega b E_{m}^{-1}\right)=\eta_{b}^{-1}\left(1-i\left(\omega_{b} / \omega_{g b}^{\delta}\right) \omega^{\delta-1}\right)
$$

where

$$
\omega_{b}=E_{m} / a b \eta_{0 b}
$$

Since $\delta<1$, at very high frequencies,

$$
\omega \gg\left(\omega_{b} / \omega_{g \mathrm{~b}}^{\delta}\right)^{(1 /(1-\delta))}
$$

the imaginary part of $F_{b m}$ tends to vanish, so that

$$
F_{b m} \cong \eta_{b}
$$

and hence

$$
F^{-1}=\eta_{g}^{-1} \cdot \frac{\left\{2(1-\gamma) \eta_{g}^{-1}+(1+2 \gamma) \eta_{b}^{-1}\right\}}{\left\{(2+\gamma) \eta_{g}^{-1}+(1-\gamma) \eta_{b}^{-1}\right\}}
$$

The friction coefficient of the syncytium given by the expression (41) (as a whole) leads to the US attenuation that reads

$$
2 \alpha_{t} / k=-\left(c_{t} / c_{f t}\right)^{2} \omega\left(1-\beta_{e}\right)(1+\varepsilon \gamma) \rho_{11} \operatorname{Re}\left\{F^{-1}\right\}
$$

where $\varepsilon$ represents the normalized difference of water content between the cells and the extra-cellular matrix that reads

$$
\varepsilon=\left(\beta_{e}-\beta_{c}\right) /\left(1-\beta_{e}\right)
$$

It is useful to note that for $\beta_{\mathrm{e}}$ close to $1, \varepsilon$ can be nega- 
tive and even bigger than one.

In Figure 1 it is shown the change of the normalized US attenuation as a function of the fraction of the cell volume $\gamma$ for two values of the permeability ratio between cell body and the extra-cellular matrix: $\eta_{b} / \eta_{g}=$ 0.01 and $\eta_{b} / \eta_{g}=0.1$.

In the case $\eta_{b} \ll \eta_{g}$, the overall friction coefficient reads

$$
F^{-1}=\eta_{g}^{-1}[(1+2 \gamma) /(1-\gamma)]
$$

while for $\eta_{b} \gg \eta_{g}$ reads

$$
F^{-1}=\eta_{g}^{-1}[(1-\gamma) /(1+\gamma / 2)]
$$

When the cellular volume is a small part of the total volume of the tissue $(\gamma \ll 1)$ it follows that

$$
F^{-1}=\eta_{g}^{-1}\left\{1-\gamma\left(2 \eta_{g}^{-1}-3 \eta_{b}^{-1}\right) /\left(2 \eta_{g}^{-1}+\eta_{b}^{-1}\right)\right\}
$$

That for $\eta_{b} \ll \eta_{g}$ leads to

$$
F^{-1}=\eta_{g}^{-1}(1+3 \gamma),
$$

while for $\eta_{b} \gg \eta_{g}$ gives

$$
F^{-1}=\eta_{g}^{-1}(1-3 \gamma / 2)
$$

In Figure 2 it is shown the change of the normalized US attenuation as a function of the fraction of the cell volume $\gamma$ for three values of the permeability ratio: $\eta_{b} / \eta_{g}=100, \eta_{b} / \eta_{g}=10 \quad \eta_{b} / \eta_{g}=2$.

In Figure 3 it is shown the US attenuation for a value of $\gamma=0.80$ typical of the liver tissue as a function of the permeability ratio $\eta_{b} / \eta_{g}$ varying from $10^{-2}$ to 100 .

By comparing formula (47) with (48) we can see that a very different behavior for the US attenuation happens whether or not $\eta_{b}$ is bigger than $\eta_{g}$. The angular coefficient of the $\gamma$-linear relations $(47,48)$ changes from positive $(+3)$ for $\eta_{b} \ll \eta_{g}$, to negative $(-3 / 2)$ for $\eta_{b} \gg \eta_{g}$

\section{Experimental}

\subsection{Materials and Methods}

Gel samples were prepared dissolving $0.5 \mathrm{ml}$ of an aqueous solution of sodium alginate at a concentration of $2 \%$ by weight (Alginic acid sodium salt from brown algae, Sigma A0682-1006) in $0.5 \mathrm{ml}$ of $\mathrm{CaCl}_{2}$ solution (FLUKA 06991 ) at a concentration of $0.4 \%$ by weight to obtain the cross-liking of the polymer matrix.

The gel samples were refrigerated at $-20^{\circ} \mathrm{C}$ for $24 \mathrm{~h}$ and then lyophilized at $-40^{\circ} \mathrm{C}$ under vacuum for 12 hours.

The gel samples were inseminated by liver cells at various densities: $10^{5}$ cells $/ \mathrm{cm}^{3}, 2 \times 10^{5}$ cells $/ \mathrm{cm}^{3}, 5 \times 10^{5}$ cells $/ \mathrm{cm}^{3}, 10^{6}$ cells $/ \mathrm{cm}^{3}, 2 \times 10^{6}$ cells $/ \mathrm{cm}^{3}, 5 \times 10^{6}$ cells $/ \mathrm{cm}^{3}$,

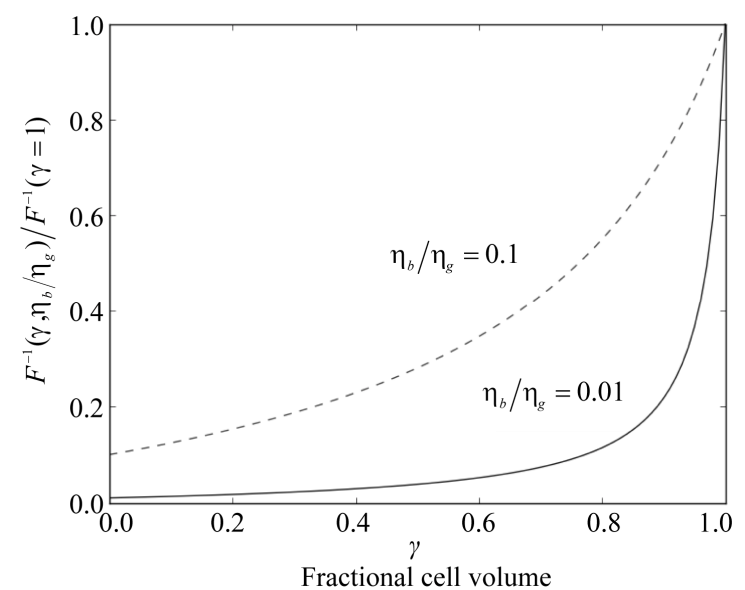

Figure 1. Change of the normalized US attenuation as a function of the fraction of the cell volume $\gamma$ for two values of the permeability ratio: $\eta_{b} / \eta_{g}=0.01$ and $\eta_{b} / \eta_{g}=0.1$.

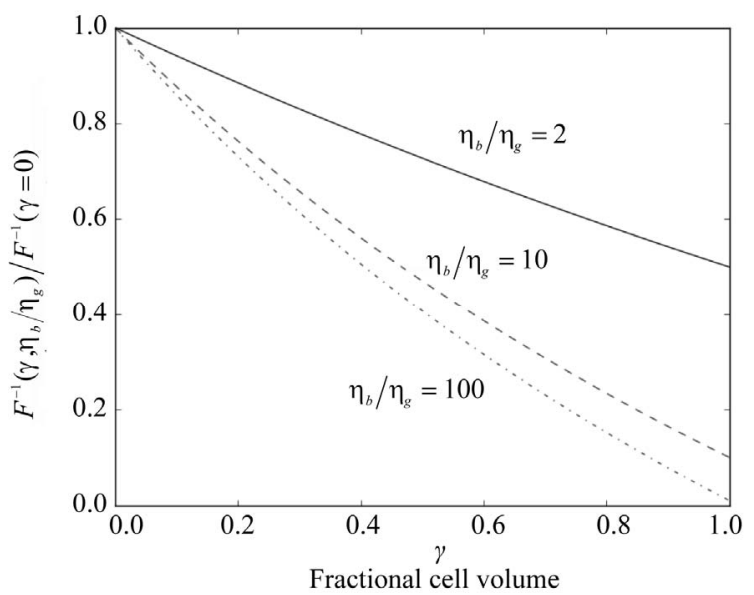

Figure 2. Change of the normalized US attenuation as a function of the fraction of the cell volume $\gamma$ for the values of the permeability ratio: $\eta_{b} / \eta_{g}=100, \eta_{b} / \eta_{g}=10, \eta_{b} / \eta_{g}=2$.

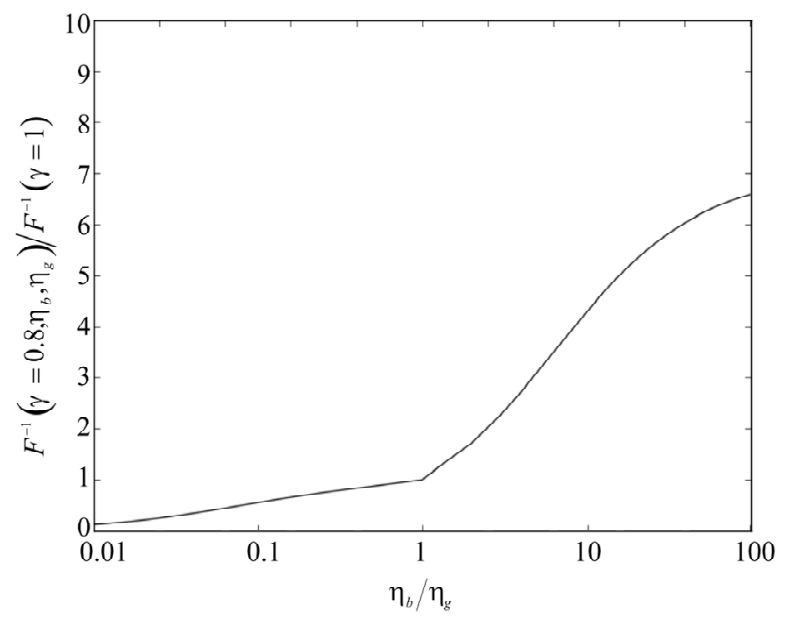

Figure 3. US attenuation for $\gamma=\mathbf{0 . 8 0}$ as a function of the permeability ratio $\eta_{b} / \eta_{g}$ varying from $10^{-2}$ to 100 . 
placed into an incubator at for 30 minutes and then in a refrigerator at $4^{\circ} \mathrm{C}$.

The ultrasonic pulses were generated by the Panametrics ${ }^{\circledR}$ Pulser model 5052PR coupled with a PVDF piezoelectric transducer obtained in our laboratory following the Naganishi e Ohigashi procedure [25].

The frequencies of the ultrasonic wave used in the experimental test were of $1.4 \mathrm{MHZ}$ and $1 \mathrm{MHz}$. The distance between the transducer and the reflecting iron layer behind the samples was measured with an accuracy of $\pm 0.01 \mathrm{~cm}$.

Echo Signal registration and conditioning data were collected with a routine and carried out with the LabView ${ }^{\mathrm{TM}}$ software on a computer through a National Instruments ${ }^{\circledR}$ DAQ device.

Finally, the samples were totally dehydrated in an oven at $40^{\circ} \mathrm{C}$ with desiccant silica gels, to measure their polymer content and the US speed in the dry solid.

The US absorption coefficient " $\alpha$ " was deduced by using the mathematical relation $\alpha=\frac{1}{2 d} \ln \frac{A_{0}}{A_{(2 d)}}$, where $A_{0}$ and $A_{(2 d)}$ represent both the initial and final wave amplitude, respectively, and where $d$ is the sample thickness.

The water volume fraction of the hydrogel samples $\beta=V_{w} /\left(V_{w}+V_{p}\right)$, where $V_{w}$ and $V_{p}$ are the volume of water and polymer respectively, was obtained by means of the respective weight fractions $P_{w}$ and $P_{p}$ such as $\beta=P_{w} /\left(P_{w}+P_{p}\right)$ since the water and PVA specific densities are very close each other.

The fitting of the experimental results were carried out by means of a multiple parameter best fit utilizing an appropriate routine in MATLAB ${ }^{\circledR}$ 7.0.

\subsection{Measurement of Ultrasound Wave Attenuation}

Introducing the measured values $\beta_{e}=0.9$ for our extracellular PVA scaffold and $\beta_{c}=0.89$ for the cells in (43), we obtain $\varepsilon \cong 0.1$.

By introducing this value in Equation (42), the bestfitted curve of experimental US attenuation in Figure 4 has been obtained for the ratio $\eta_{b} / \eta_{g}=6.54$.

The result put in evidence that once the permeability of the extra-cellular gel scaffold $\eta_{g}^{-1}$ is known or measured, the US poro-elastic model allows deriving the permeability of the cellular bulk $\eta_{b}^{-1}$.

\section{Discussion}

The acoustic bi-phasic model for soft living tissues describes the US propagation in terms of collective cells and extra-cellular matrix characteristics such as: 1) The permeability and the elasticity of the cells and of the extra-

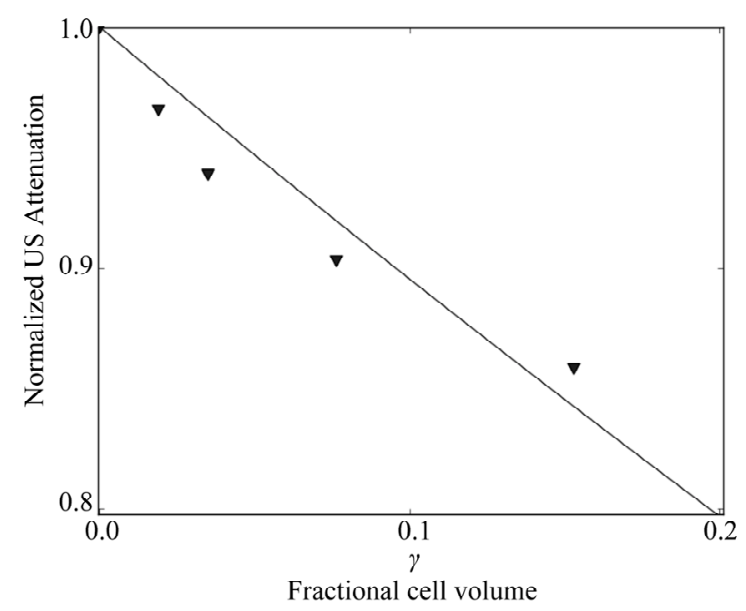

Figure 4. Experimental values of the normalized US attenuation in PVA-porcine liver cells composites with the best fit obtained for $\eta_{b} / \eta_{g}=6.54$ as a function of the fraction of the cell volume $\gamma$.

cellular matrix; 2) The percentage of cellular volume of the tissue; 3 ) The fractional volume of water of cells and of the extra-cellular matrix.

As far as it concerns the wave speed, the model does not make an explicit derivation of the coefficients $A_{1}$ and $\mathrm{A}_{2}$ by the constituents of the cellular syncytium. On the contrary, the model details how the US attenuation depends by the cell elasticity and permeability through the term $\operatorname{Re}\left\{F^{-1}\right\}$.

By using this information, it is possible to define an experimental method for the measurement of the permeability of the cells once that one of the external scaffold is known.

Moreover, since the poro-elastic characteristics of the cells and extra-cellular matrix may appreciably change in pathological states (e.g. as in the cirrhosis) in principle, the health state of biological tissues can be tracked by means of ultrasounds.

In particular, the shape of the absorbance frequency spectrum determined by the characteristic frequencies

$$
\begin{gathered}
\omega_{g b}=2 \pi \eta_{0 b} / \rho_{f b}, \\
\omega_{b}=E_{m} / a b \eta_{0 b}
\end{gathered}
$$

and

$$
\omega_{m}=K_{m} E_{m} b^{-1}
$$

can give information about the health state of cells and tissues (as a sort of eco-biopsy) on the basis of epidemiological comparisons.

\section{Concluding Remarks}

The bi-phasic continuum model for US propagation in hydrogels has been used to build up the acoustic wave 
equation for a tissue-like syncytium made of spherical cells homogeneously immersed in an extra-cellular gel matrix.

The model shows that the absorption of US is sensitive to the cellular content of the tissue as well as of the porosity of the cells body with respect to the external matrix.

The model shows that the spectrum of US absorption in a biological tissue has a characteristic shape depending by the elasticity and permeability of cells and extracellular matrix. By means of these parameters that are linked to the health state of a tissue, the model can be used to monitor pathologies of it.

The model agrees with preliminary measurements done on porcine liver cells embedded in a poly-vinylalcohol matrix. The experimental results have put in evidence that the porcine liver cells have the bulk permeability lower than that one of the PVA gel scaffold.

\section{References}

[1] F. A. Duck, "Acoustic Properties of Tissue at Ultrasonic Frequencies," Academic Press, London, New York, 1990, pp. 75-99.

[2] F. W. Kremkau, R. W. Barnes and C. P. McGraw, "Ultrasonic Attenuation and Propagation Speed in Normal Human Brain," Journal of Acoustical Society of American, Vol. 70, No. 1, 1981, pp. 29-38. doi: $10.1121 / 1.386578$

[3] J. W. Wladimiroff, I. L. Craft and D.G. Talbert, "In Vitro Measurements of Sound Velocity in Human Fetal Brain Tissue," Ultrasound in Medicine \& Biology, Vol. 1, No. 4, 1975, pp. 377-382. doi:10.1016/0301-5629(75)90125-8

[4] P. Chiarelli, et al., "High Frequency Poroelastic Waves in Hydrogel,s" Journal of Acoustical Society of American, Vol. 127, No. 3, 2010, pp. 1197-1207. doi: $10.1121 / 1.3293000$

[5] D. De Rossi, A. Nannini and C. Domenici, "Artificial Sensing Skin Mimicking Mechanoelectrical Conversion Properties of Human Dermis," IEEE Transaction on Biomedical Engineering, Vol. 35, No. 8, 1988, pp. 3-92. doi:10.1109/10.1343

[6] S. Lochhead, D. Bradwell, R. Chopra and M. J. Bronskill, "A Gel Phantom for the Calibration of MR-Guided Ultrasound Thermal Therapy," Proceedings of 2004 IEEE Ultrasonics Symposium, Montreal, Vol. 2, 23-27 August 2004, pp. 1481-1483.

[7] G. Divkovic, M. Liebler, K. Braun, T. Dreyer, P. Huber and J. Jenne, "Thermal Properties and Changes of Acoustic Parameters in an Egg White Phantom during Heating and Coagulation by High Intensity Focused Ultrasound," Ultrasound in Medicine Biology, Vol. 33, No. 6, 2007, pp. 981-986.

[8] M. Ziol, et al., "Noninvasive assessment of Liver Fibrosis by Measurement of Stiffness in Patient with Chronic Hepatitis C," Hepatology, Vol. 41, No. 1, 2005, pp. 48-54. doi:10.1002/hep.20506

[9] G. P. Berry, J. C. Bamber, C. G. Armstrong, N. R. Miller and P. E. Barbonne, "Toward an Acoustic Model-Based Poroelasticity Imaging Method: I. Theoretical Foundation," Ultrasound in Medicine Biology, Vol. 32, No. 4, 2006, pp. 547-567.

doi:10.1016/j.ultrasmedbio.2006.01.003

[10] J. Bercoff, M. Tanter and M. Fink, "Supersonic Shear Imaging: A New Technique for Soft Tissue Elasticity Mapping," IEEE Transactions on Ultrasonics, Ferroelectrics and Frequency Control, Vol. 51, No. 4, 2004, pp. 396-409. doi:10.1109/TUFFC.2004.1295425

[11] M. L. Mather and C. Baldock, "Ultrasound Tomography Imaging of Radiation Dose Distributions in Polymer Gel Dosimeters: Preliminary Study," Medical Physics, Vol. 30, No. 8, 2003, pp. 2140-2148. doi:10.1118/1.1590751

[12] X. Yang and R. O. Cleveland, "Time Domain Simulation of Nonlinear Acoustic Beams Generated by Rectangular Piston with Application to Harmonic Imaging," Journal of Acoustical Society of American, Vol. 171, No. 1, 2005, pp. 113-123.

[13] J. C. Bacri, J. M. Courdille, J. Dumas and R. Rajaonarison, "Ultrasonic Waves: A Tool for Gelation Process Measurements," Journal of Physique Letters, Vol. 41, No. 15, 1980, pp. 369-372.

[14] F. A. Duck, "Acoustic Properties of Tissue at Ultrasonic Frequencies," Academic Press, London, New York, 1990, pp. 112-113.

[15] M. A. Biot, "General Theory of Three-Dimensional Consolidation," Journal of Applied Physics, Vol. 12, No. 2, 1941, pp. 155-164. doi:10.1063/1.1712886

[16] M. A. Biot, "Theory of Propagation of Elastic Waves in a Fluid-Saturated Porous Solid. II. High Frequency Range," Journal of Acoustical Society of American, Vol. 28, No. 2, 1956, pp. 179-191.

[17] M. A. Biot, "Theory of Propagation of Elastic Waves in a Fluid-Saturated Porous Solid. I. Low-Frequency Range," Journal of Acoustical Society of American, Vol. 28, No. 2, 1956, pp. 168-178.

[18] M. A. Biot, "The Elastic Coefficients of the Theory of Consolidation," Journal of Applied Mechanics, Vol. 24, No., 1957, pp. 594-601.

[19] D. L. Johnson, "Elastodynamics of Gels," Journal of Chemical Physics, Vol. 77, No. 3, 1982, pp. 1531-1539. doi: $10.1063 / 1.443934$

[20] R. N. Chandler, "Transient Streaming Potential Measurements on Fluid-Saturated Porous Structures: An Experimental Verification of Biot's Slow Wave in the QuasiStatic Limit," Journal of Acoustical Society of America, Vol. 70, No. 1, 1981, pp. 116-121. doi:10.1121/1.386689

[21] A. Peters and S. J. Candau, "Kinetics of Swelling of Spherical and Cylindrical Gels," Macromolecules, Vol. 21, No. 7, 1988, pp. 2278-2282. doi:10.1021/ma00185a068

[22] D. L. Johnson, "Equivalence between Fourth Sound in Liquid He II at Low Temperature and the Biot Slow Wave in Consolidated Porous Media," Applied Physics Letters, Vol. 37, No. 12, 1980, pp. 1065-1067. 
doi:10.1063/1.91878

[23] J. G. Berryman, "Confirmation of Biot's Theory," Applied Physics Letters, Vol. 37, No. 4, 1980, pp. 382-384. doi:10.1063/1.91951

[24] B. J. Roth, "The Electrical Conductivity of Tissues," In: J.
D. Bronzino, Ed., The Biomedical Engineering Handbook, 2nd Edition, CRC Press LLC, Boca Raton, 2000.

[25] T. Naganishi, M. Suzuki and H. Ohigashi, "Ultrasonic Transducers," United States Patent No. 4.296.349, 1981. 